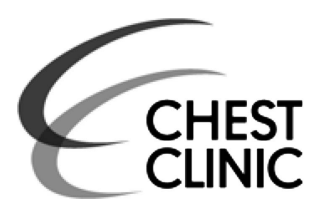
Medicine, Royal Infirmary of Edinburgh, Edinburgh, UK

${ }^{2}$ MRC Centre for Inflammation Research, Queen's Medical Research Institute, Edinburgh, UK

${ }^{3}$ The British Thoracic Society, London, UK

\section{Correspondence to}

Dr Adam Hill, Department of Respiratory Medicine, Royal Infirmary of Edinburgh, 51 Little France Crescent, Old Dalkeith Road,

Edinburgh EH16 4SA, UK; adam.hill318@nhs.net

Received 15 April 2013 Revised 28 May 2013 Accepted 1 July 2013 Published Online First 22 July 2013

\begin{tabular}{l}
\hline To cite: Hill AT, Routh C, \\
Welham S. Thorax \\
2014;69:292-294.
\end{tabular}

\title{
National BTS bronchiectasis audit 2012: is the quality standard being adhered to in adult secondary care?
}

\author{
Adam T Hill, ${ }^{1,2}$ Chris Routh, ${ }^{3}$ Sally Welham ${ }^{3}$
}

\begin{abstract}
A significant step towards improving care of patients with non-cystic fibrosis bronchiectasis was the creation of the British Thoracic Society (BTS) national guidelines and the quality standard. A BTS bronchiectasis audit was conducted between 1 October and 30 November 2012, in adult patients with bronchiectasis attending secondary care, against the BTS quality standard. Ninety-eight institutions took part, submitting a total of 3147 patient records. The audit highlighted the variable adoption of the quality standard. It will allow the host institutions to benchmark against UK figures and drive quality improvement programmes to promote the quality standard and improve patient care.
\end{abstract}

\section{INTRODUCTION}

There has been a significant development in the standardisation of bronchiectasis management in the past 3 years. The British Thoracic Society (BTS) published guidelines for non-cystic fibrosis (CF) bronchiectasis in July 2010 and developed the quality standard for non-CF bronchiectasis in adults in 2012. ${ }^{12}$ A national BTS bronchiectasis audit was conducted between 1 October and 30 November 2012, in adult patients with bronchiectasis attending secondary care, against the BTS quality standard.

\section{PATIENT PROFILE}

In keeping with international studies the majority of patients were female $(60 \%)$ with a mean age of $66 \pm 13$ years. ${ }^{13-5}$ From the sputum microbiology in the preceding year Psendomonas aeruginosa, methicillin-resistant Staphylococcus aureus or enteric Gram-negative organisms (the last of these if isolated on two or more occasions (eg, Escherichia coli)) were isolated in 27\%; no data were obtained in $8 \%$.

The BTS guidelines recommend considering giving long-term antibiotics to patients having three or more exacerbations annually. ${ }^{1}$ In this audit $38 \%$ had three or more exacerbations a year; results for $17 \%$ were not known. Thirty-three per cent were receiving long-term antibiotic treatment either orally or by inhalation. Thirty per cent had received long-term oral antibiotics for $>28$ days; data for $1 \%$ not known. Of those receiving oral antibiotics, $69 \%$ were taking a long-term macrolide (63\% azithromycin, 4\% clarithromycin and 2\% erythromycin). There is growing evidence for the use of macrolide antibiotics as long-term anti-inflammatory treatment in bronchiectasis to reduce exacerbations. ${ }^{3-5}$ Ten per cent had received long-term inhaled antibiotics for $>28$ days; data for $2 \%$ not known. Of those receiving inhaled antibiotics, $86 \%$ were on nebulised Colomycin, 6\% gentamicin, $4 \%$ tobramycin and $4 \%$ others.

\section{AUDIT OF THE QUALITY STANDARD}

The quality standard comprises 11 quality statements (table 1). The statements are in no particular order of importance but all aim to improve the management of patients in primary and secondary care. The audit included 98 secondary care institutions throughout the UK and 3147 records were submitted during the audit period.

The first quality statement recommends ensuring diagnostic accuracy with confirmation of a clinical diagnosis of bronchiectasis with a CT of the chest using $1 \mathrm{~mm}$ slices. In this audit, $91 \%$ had a CT scan of the chest, $0.3 \%$ were diagnosed by bronchography, $2.7 \%$ had a clinical diagnosis alone and results for $6 \%$ were not known. This quality statement aims to promote accurate radiological confirmation of a clinical diagnosis of bronchiectasis using modern imaging to define patients in primary and secondary care. It is well recognised that reliance on plain chest radiography may lead to both an underdiagnosis and overdiagnosis of bronchiectasis. ${ }^{1}$

The second statement proposes that people with bronchiectasis are taught appropriate airway clearance techniques by a specialist respiratory physiotherapist and advised of the frequency and duration with which these should be carried out. Seventy-eight per cent were taught chest clearance techniques by a specialist respiratory physiotherapist, $12 \%$ were not and for $10 \%$ no data were available. The data from this audit did not show how often patients were followed up by the respiratory physiotherapist. A considerable number of patients are still not being given the recommended treatment-a key treatment for alleviating symptoms and which may reduce chest infections.

The third statement requires that people with bronchiectasis have sputum bacteriology culture when clinically stable recorded at least once each year. Sixty-two per cent had this culture, 22\% did not, for $8 \%$ it was not applicable and for $8 \%$ details were not known. The fourth statement was that sputum be sent for bacterial culture at the start of an exacerbation before starting antibiotics. Empirical antibiotic treatment should start as soon as feasible and not await the sputum culture results. 
Table 1 Summary of the quality statements and percentage adherence

\section{Quality statements for clinically significant bronchiectasis in adults}

People with a clinical diagnosis of bronchiectasis have the diagnosis confirmed by chest CT scan (using $1 \mathrm{~mm}$ slices)

People with bronchiectasis are taught appropriate airway clearance techniques by a specialist respiratory physiotherapist and advised of the frequency and duration with which these should be carried out

People with bronchiectasis have sputum bacteriology culture when clinically stable recorded at least once each year

Sputum is sent for bacterial culture at the start of an exacerbation before starting antibiotics. Empirical antibiotic treatment to start as soon as feasible and 53 not await the sputum culture results

People with bronchiectasis to attend pulmonary rehabilitation if they have breathlessness affecting their activities of daily living

People with bronchiectasis receiving intravenous antibiotic treatment to have an objective evaluation of the efficacy of their treatment and the result recorded

Services for people with bronchiectasis to include provision of nebulised prophylactic antibiotics for suitable patients supervised by a respiratory specialist

Fifty-three per cent had sputum sent for bacterial culture at the appropriate time, $17 \%$ did not, for $15 \%$ it was not applicable and for $15 \%$ details were not known. The third and fourth statements aim at improving basic microbiology surveillance to help guide antibiotic treatment and management and improve the assessment and follow-up.

The fifth statement proposes that people with bronchiectasis and breathlessness affecting their activities of daily living should attend pulmonary rehabilitation. Fifty-four per cent had breathlessness, $17 \%$ did not, for $15 \%$ it was not applicable and for $15 \%$ details were not known. Of those affected by breathlessness, 32\% attended a pulmonary rehabilitation programme, $51 \%$ did not and for $17 \%$ the information was not known. The audit required reasons for non-attendance at a pulmonary rehabilitation programme. These were as follows: in $42 \%$ the patient was not referred, $18 \%$ were not fit owing to a comorbid illness, $14 \%$ refused, $12 \%$ were on the waiting list for rehabilitation, for $10 \%$ no rehabilitation programme was available, for $3 \%$ the reason was not known and $1 \%$ just did not attend. The benefits of pulmonary rehabilitation are to improve patients exercise capacity and health status.

The sixth statement is that people with bronchiectasis receiving intravenous antibiotic treatment should have an objective evaluation of the efficacy of their treatment and the result recorded. Eighteen per cent had required intravenous antibiotics in the preceding year. Of these, $78 \%$ had an objective evaluation at the beginning and end of treatment. The most common assessments were sputum colour $(83 \%)$, sputum volume $(70 \%)$, C-reactive protein $(81 \%)$, oxygen saturations on room air $(71 \%)$, white cell count $(77 \%)$ and sputum bacteriology (71\%). Spirometry was carried out in 32\%. Assessments at the beginning and end of treatment will allow the patient and clinicians to assess objectively the response and may guide long-term management.

The seventh statement requires that there should be provision of nebulised prophylactic antibiotics for suitable patients supervised by a respiratory specialist. Ten per cent were receiving long-term nebulised antibiotics for $>1$ month. Of these, $86 \%$ were receiving nebulised Colomycin, $6 \%$ gentamicin, $4 \%$ tobramycin and $4 \%$ others. Ninety-four per cent of trusts have facilities to offer domiciliary inhaled antibiotic treatment. Long-term prophylactic treatment may improve symptoms and reduce the number of chest infections. ${ }^{1}$
The eighth statement recommends that people with bronchiectasis should be investigated for allergic bronchopulmonary aspergillosis (ABPA), common variable immunodeficiency (CVID) and CF (the last of these if indicated) as these are specific treatable causes. In this audit we did not ask what specific tests were carried out. Sixty-three per cent had been investigated for ABPA, $26 \%$ had not, for $9 \%$ details were not known and for $1 \%$ it was not applicable. Sixty-eight per cent had been investigated for CVID, 22\% had not, for 9\% details were not known and for $1 \%$ it was not applicable. Twelve per cent had been investigated for CF, $41 \%$ had not, for $18 \%$ details were not known and for $29 \%$ it was not applicable. This statement is important as these causes of bronchiectasis have specific treatments that differ from standard bronchiectasis management, which may alter the prognosis.

The ninth statement is that people with bronchiectasis should have an individualised written self-management plan. Thirty-three per cent had, $45 \%$ did not and for $22 \%$ it was not known. This will allow people with bronchiectasis to manage their condition and to recognise, respond to, and reduce, the occurrence of chest infections. A sample self-management plan is on the BTS website and can be modified for local use. ${ }^{6}$

The tenth standard is that people with bronchiectasis who meet the criteria for continuing secondary care should be managed by a multidisciplinary team led by a respiratory physician. $^{1}$ Of the conditions listed in the guideline for follow-up (figure 1), 23\% had chronic Pseudomonas aeruginosa infection, opportunistic mycobacteria or methicillin-resistant $S$ aureus infection. Ten per cent had deteriorating bronchiectasis with

\footnotetext{
Patients who should have regular follow-up in secondary care include:

- all children with bronchiectasis

- patients with chronic Pseudomonas aeruginosa, opportunist mycobacteria or methicillin-resistant Staphylococcus aureus colonisation:

- deteriorating bronchiectasis with declining lung function;

- recurrent exacerbations ( 3 or more per year);

- patients receiving prophylactic antibiotic therapy (oral or nebulised);

- patients with bronchiectasis and associated rheumatoid arthritis, immune deficiency, inflammatory bowel disease and primary ciliary dyskinesia;

- patients with ABPA;

- patients with advanced disease and those considering transplantation.
}

Figure 1 Patients who should have regular follow-up in secondary care. ABPA, allergic bronchopulmonary aspergillosis. 
declining lung function, $42 \%$ had three or more exacerbations a year, 33\% were receiving long-term antibiotic treatment either oral or inhaled and $13 \%$ had bronchiectasis associated with rheumatoid arthritis, immune deficiency, inflammatory bowel disease or primary ciliary dyskinesia. Finally, 7\% had ABPA and $3 \%$ had advanced disease and/or were considering lung transplantation. The percentages add up to more than $100 \%$ as some patients had a combination of factors.

Of these conditions listed in the guidelines for follow-up in secondary care at least 79 out of 84 trusts would organise follow-up in their trusts. ${ }^{1}$ Of those attending secondary care, $41 \%$ were seen every $2-3$ months, $34 \%$ were seen 6 monthly, $8 \%$ less often, for $14 \%$ details were not known and $3 \%$ were not receiving active follow-up. In people with non-standard bronchiectasis, the clinical course and management can be complicated and would be better managed by a multidisciplinary team led by a respiratory physician. ${ }^{1}$

The final statement concerns services for people with bronchiectasis which should include provision of home intravenous antibiotic treatment for exacerbations in selected patients. In the audit $40 \%$ were offered domiciliary intravenous antibiotics, $21 \%$ were not, $19 \%$ were not suitable, for $10 \%$ data were not available and for $10 \%$ the information was not known. The aim of domiciliary intravenous treatment is to reduce hospital bed days and the risk of hospital-acquired infection, while promoting people-centred care by allowing delivery of intravenous treatment safely at home. Seventy-one per cent of trusts can offer this service but $27 \%$ cannot and $2 \%$ did not know.

\section{CONCLUSIONS}

This UK national audit has shown that response to the quality standard, which was created to deliver standardised high-quality care, is variable. Five areas requiring particular attention include: ensuring investigation for ABPA, CVID and CF; monitoring sputum bacteriology; promoting pulmonary rehabilitation in patients with breathlessness affecting their activities of daily living; developing services to allow domiciliary intravenous antibiotic treatment and, finally, providing a written selfmanagement plan for all patients. We encourage trusts to use the results of the 2012 audit and the BTS quality standard to set in place quality improvement measures to deal with the areas identified in the audit as requiring attention and ensure that the audit cycle is completed.

Contributors ATH: conception and design of the quality standard as well as the analysis and interpretation of data. He drafted and revised the article and gave final approval for publication. CR: design and collection of data and presentation of the article, revision of the article and final approval. SW: involved in all stages from the conception and design of the quality standard, collection and analysis of data, drafting the revision of the article and final approval.

Competing interests None.

Provenance and peer review Not commissioned; externally peer reviewed.

\section{REFERENCES}

1 Pasteur MC, Bilton D, Hill AT; British Thoracic Society Bronchiectasis non-CF Guideline Group. British Thoracic Society guideline for non-CF bronchiectasis. Thorax 2010;65(Suppl 1):i1-58. Review.

2 Hill AT, Bilton D, Brown J, et al. BTS Quality Standards for clinically significant bronchiectasis in adults. Br Thorac Soc Rep 2012;4:1-16. ISSN: 2040-2023. http:// www.brit-thoracic.org.uk/Portals/0/Guidelines/Bronchiectasis/244457_BTS_Quality_ Standards_Bronchiectasis.pdf

3 Wong C, Jayaram L, Karalus N, et al. Azithromycin for prevention of exacerbations in non-cystic fibrosis bronchiectasis (EMBRACE): a randomised, double-blind, placebo-controlled trial. Lancet 2012;380:660-7.

4 Serisier DJ, Martin ML, McGuckin MA, et al. Effect of long-term, low-dose erythromycin on pulmonary exacerbations among patients with non-cystic fibrosis bronchiectasis: the BLESS randomized controlled trial. JAMA 2013;309:1260-7.

5 Altenburg J, de Graff CS, Stienstra Y, et al. Effect of azithromycin maintenance treatment on infectious exacerbations among patients with non-cystic fibrosis bronchiectasis: the BAT randomized controlled trial. JAMA 2013;309:1251-9.

6 BTS self management plan. http://www.brit-thoracic.org.uk/Portals/0/Guidelines/ Bronchiectasis/BTS\%20Bx\%20SMP\%2029\%20Feb\%20website.pdf 